\title{
Consumo de leña y estimaciones del valor calorífico de especies de plantas utilizadas por las comunidades campesinas de la cordillera del Vilcanota, Cusco
}

\section{Consumption of firewood and calorific value estimation of plant species used by farmers communities of the cordillera Vilcanota, Cusco}

\author{
Juan E. Gil-Mora ${ }^{1}$, Jesús A. Baca-Flores ${ }^{2} \&$ Modesta E. Álvarez-Moscoso ${ }^{3}$
}

'Escuela de Posgrado, Universidad Nacional de San Antonio Abad del Cusco. Av. de la Cultura, 733, Cusco, Perú.E-mailmundoandino2005@yahoo.es

${ }^{2}$ Departamento Académico de Física; Facultad de Ciencias. Universidad Nacional San Antonio Abad del Cusco. Av. de la Cultura, 733, Cusco, Perú

${ }^{3}$ Escuela Profesional de Biología; Facultad de Ciencias. Universidad Nacional San Antonio Abad del Cusco. Av. de la Cultura, 733, Cusco, Perú

\section{Resumen}

A pesar de que la vegetación arbórea y arbustiva leñosa se halla intervenida y deteriorada en la zona altoandina, existen especies como fuentes de energía. El estudio se desarrolló en 12 comunidades campesinas localizadas en las microcuencas de los ríos Patacancha (Ollantaytambo-Urubamba) y Lares (Lares-Calca); han sido identificadas 56 especies nativas empleadas como leña; estas especies son utilizadas por las comunidades campesinas ubicadas por encima de 3,200 msnm en la cordillera del Vilcanota. El requerimiento comunal de leña y energía es de $1.754 \mathrm{~kg}$. de combustible por persona al día; esto implica un consumo en valores de energía de 12,170.88 kJ/ kg, lo que significa un consumo de 0.0227 GJ persona/día. El $62.71 \%$ del combustible utilizado por las comunidades altoandinas proviene de bosques nativos, el $13.97 \%$, de la bosta de ganado y el 23.3\% del eucalipto. Si esta demanda es comparada con la oferta de leña existente en los bosques nativos, se concluye que existe un déficit de oferta de energía por parte de la biomasa vegetal existente en los bosques montanos altoandinos. Se han identificado especies de interés para la recuperación de los bosques altoandinos, como las que pertenecen a los géneros: Polylepis, Escallonia, Hesperomeles, Duranta, Citharexylum, Vallea, Berberis, Myrcianthes que poseen un alto poder calorífico que fluctúa entre 11,500 y $12,500 \mathrm{KJ} / \mathrm{kg}$.

Palabras clave. Bosque montano, bosta, densidad, energía, humedad.

\section{Abstract}

Despite the fact that the woody and shrub vegetation in the high Andean zone is intervened and therefore deteriorated, there still are species as energy sources. The study was carried out in 12 peasant communities located in the micro-basins of the Patacancha (Ollantaytambo-Urubamba) and Lares (LaresCalca) rivers. There had been identified 56 native species used as firewood; which are used by peasant communities located above 3200 masl in the Vilcanota mountain range. Community firewood and energy requirement is $1.754 \mathrm{~kg}$ of fuel per person per day; implying values of energy consumption of 12,170.88 $\mathrm{kJ} / \mathrm{kg}$, meaning a consumption of $0.0227 \mathrm{GJ}$ person/day. $62.71 \%$ of the fuel used by the high Andean communities comes from native forests, $13.97 \%$ from cattle dung and $23.3 \%$ from eucalyptus. If this demand is compared with the supply of firewood existing in the native forests, it is concluded that there is a deficit of energy supply by the existing plant biomass in the high Andean montane forests. There have been identified interest species for high Andean forests recovery, such as those belonging to Polylepis, Escallonia, 
- Hesperomeles, Duranta, Citharexylum, Vallea, Berberis, Myrcianthes genus, which have a high calorific value fluctuating between 11,500 and $12,500 \mathrm{KJ} / \mathrm{kg}$.

Kew words. Density, energy, humidity, manure, montane forest

\section{Introducción}

Los bosques andinos, materia de nuestro estudio, han sido analizados por diversos autores, De Rutté (2014), señala que los bosques de neblina de la vertiente oriental de la cordillera de los Andes son ecosistemas con una diversidad biológica única; son parte de los ecosistemas más amenazados por la deforestación y el cambio climático (Kessler \& Driesch, 1993). De otro lado, el Mapa de cobertura vegetal del Perú (MINAM, 2012) a la vegetación estudiada en las comunidades altoandinas lo denomina como "bosque húmedo de montaña" que se extiende a través de todo el flanco oriental andino, desde el pie de montaña hasta aproximadamente 3,000 a 3,800 msnm y distingue tres estratos con una composición florística representativa dentro de la categoría de Bosques montanos: El piso inferior $(<1,500 \mathrm{msnm})$, piso medio $(1,500$ 2,500 o 2800) conocido como bosque de neblina y el piso superior $(>2,500$ o 2,800 msnm), como también lo especifica Portuguez et al. (2012).

Según ECOBONA (2009), una parte importante del paisaje andino lo constituye el bosque andino, que es un ecosistema frágil caracterizado por su alto grado de diversidad biológica, cuya singularidad es única. Los ecosistemas forestales andinos se encuentran entre los más diversos y amenazados ecosistemas terrestres (Toyvonen, 1990; Etter y Villa, 2000). Son reconocidos como un hotspot principal a escala global (Orme et al., 2005) y representan una prioridad para la- conservación por su extraordinaria riqueza y endemismo y porque varias de sus especies constituyentes están siendo severamente amenazadas (Aubad et al., 2008).

Según FAO y PNUMA (2020); los bosques se encuentran sujetos a numerosas perturbaciones naturales (incendios forestales, enfermedades, fenómenos meteorológicos adversos) que pueden afectar negativamente a su salud y su vitalidad al causar la mortalidad de los árboles o reducir su capacidad de proporcionar todos los bienes y servicios. Igualmente, esta entidad sostiene que la expansión agrícola sigue siendo la principal causa de la deforestación y la fragmentación de los bosques y de la pérdida de biodiversidad forestal asociada.

Tal como señala el informe de la situación en materia de leña en los países en desarrollo (FAO, 1981 y 1983), los Andes constituyen una de las regiones del planeta donde la escasez de leña es más aguda; siendo las regiones altiplánica y altoandina las que registran las mayores penurias, son en estos espacios donde los recursos de leña se han agotado a tal punto que la población ya no está en condiciones de satisfacer sus necesidades mínimas (Lara, 1982; Ocaña, 1994); análogamente a estos reportes, en las comunidades estudiadas que se hallan ubicadas por encima de los 3,600 o 4,000 msnm, el déficit de material energético es crítico y no llega a abastecer las necesidades más urgentes, especialmente si de leña se trata (Gil-Mora, 2000 y 2002); por lo tanto, es substituido por otros elementos como la bosta de ganado (vacuno, ovino y camélidos- 
sudamericanos) generados en la propia comunidad y/o la leña de eucalipto, proveniente de otras zonas.

Referido a especies andinas utilizadas por las poblaciones para satisfacer las demandas básicas, existen diversos estudios como los de Morlon (1980), Ansion y Van Dam (1986), Desarrollo forestal participativo (1992) y el de Lojan (2003) que enfocan los servicios ambientales de las especies forestales nativas, entre ellos, la leña y madera; sin embargo, no vinculan los estudios con el poder calorífico de las especies.

De otro lado, los bosques naturales en los andes son recursos renovables que pueden dar una producción permanente de bienes $\mathrm{y}$ servicios ecosistémicos (FAO, 1983; Pretel, 1985). El uso intensivo que se le da al suelo, tanto para la agricultura como para la ganadería, ha producido el empobrecimiento de los suelos y la erosión, a pesar de ello, el campesino sigue cultivando en las laderas y en suelos marginales (Geist \& Lambin, 2001).

Los bosques altoandinos, son además uno de los ecosistemas menos conocidos y más amenazados en los trópicos (Stadtmüller, 1986; Gentry, 1995; Ataroff y Rada, 2000; Kessler 2000; Bubb et al., 2004; Price et al., 2011) como consecuencia, principalmente, de las altas tasas de deforestación y degradación debidas a la expansión de actividades agropecuarias.

Las comunidades campesinas de los altos andes, ubicadas en la cordillera del Vilcanota requieren productos del bosque como fuente de energía, la leña es proveniente de bosques relictos de Escallonia, Polylepis, Gynoxys, Buddleja, Alnus, Citharexylum, Myrcianthes, Weinmannia, Myrica, Duranta, etc; esta demanda agota la oferta de bosques nativos.

La finalidad del estudio es determinar la -naturaleza de los distintos tipos de formaciones forestales y la demanda de leña como la importancia de las comunidades altoandinas dependientes de la leña, necesidades individuales de leña, estimadas a partir de la información disponible sobre el consumo energético.

La preocupación que se tiene sobre los bosques remanentes en los altos Andes, apuesta por una utilización sostenible de la vegetación; junto a los actores sociales de 12 comunidades campesinas se han identificado los problemas respecto del uso de las principales especies utilizadas como leña; se ha efectuado un análisis sobre la biomasa vegetal y la oferta de los bosques naturales de la energía disponible, calculándose la humedad y contenido calorífico de las especies utilizadas como leña y se reporta la demanda de leña utilizada por las comunidades de la cordillera del Vilcanota, donde aún existen bosques nativos.

\section{Materiales y métodos}

\section{Área de Estudio}

Las comunidades estudiadas se encuentran por encima de los 3,200 msnm en el flanco oriental de la cordillera del Vilcanota; cinco en la microcuenca del río Patacancha, distrito de Ollantaytambo, Provincia de Urubamba y siete en la microcuenca del río Lares, distrito de Lares, provincia de Calca (Figura 1). Para el estudio, estas comunidades han sido divididas en dos pisos altitudinales; aquellas ubicadas por debajo de los 3,500 msnm y otras asentadas por encima de ese nivel altitudinal. Los criterios que condujeron a esta localización altitudinal de las comunidades estudiadas, se debe, principalmente, al uso de leña y al acceso de parte de las comunidades campesinas al bosque nativo; las comunidades ubicadas por debajo 
de los 3,500 msnm cuentan con recursos forestales provenientes de los bosques nativos; en cambio, las comunidades campesinas ubicadas por encima de este nivel altitudinal, poseen escasa oferta de leña y, utilizan la takia, bosta y turba como fuentes de energía y los bosques poseen una escasa oferta de leña.

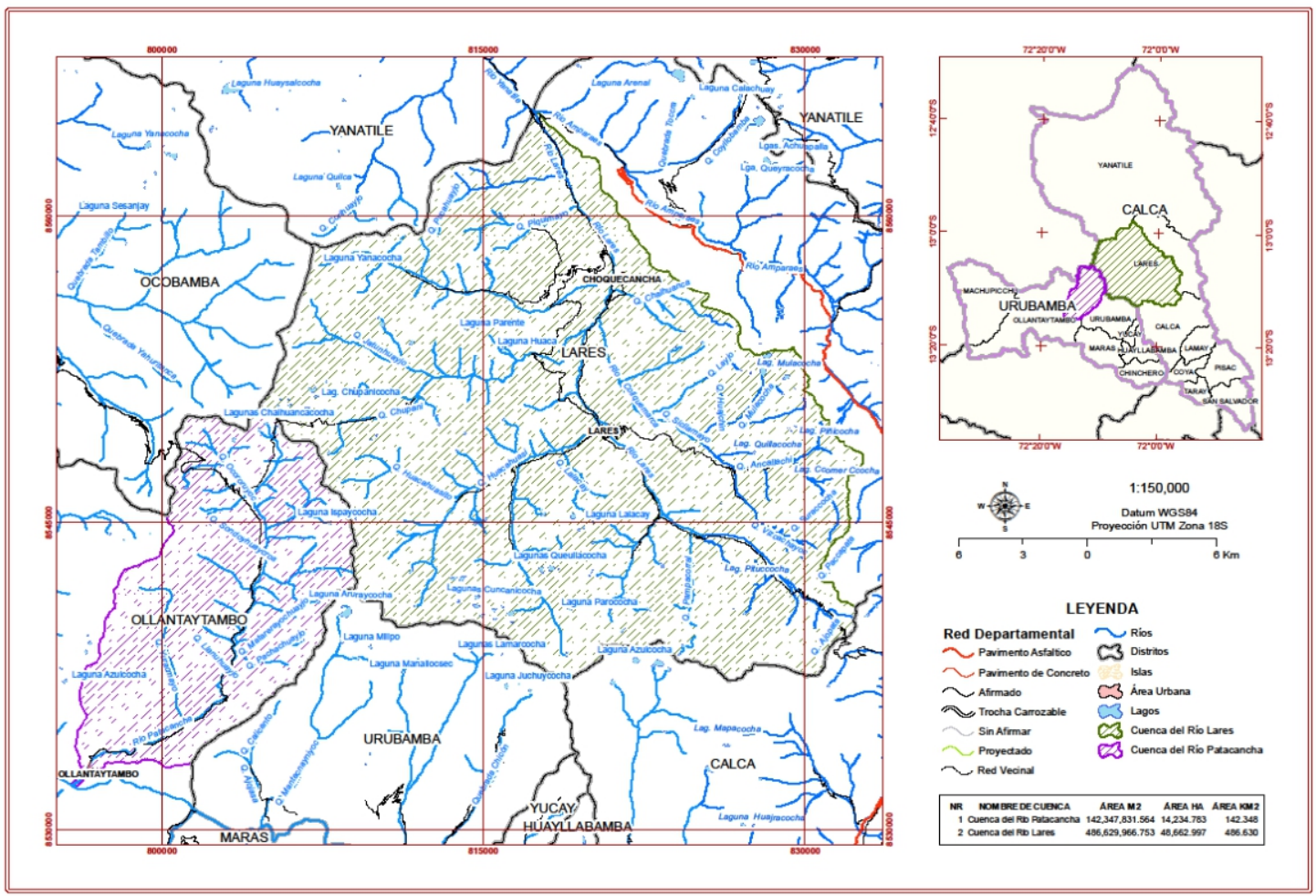

Figura 1. Ubicación de las zonas de estudio.

Tabla 1. Ubicación de las comunidades estudiadas

\begin{tabular}{|l|l|l|l|}
\hline Microcuenca & $\begin{array}{l}\text { Comunidad } \\
\text { campesina }\end{array}$ & $\begin{array}{l}\text { Altitud } \\
\mathbf{( m s n m )}\end{array}$ & Orientación referencial \\
\hline Río Málaga & T'astayoq -Abra de & 4350 & NNE respecto de Ollantaytambo \\
& Málaga & & \\
Río Patacancha & Phallata & 3,250 & ENE de Ollantaytambo \\
& Willoq & 3,950 & ENE de Ollantaytambo \\
& Rumira-Sondor & 4,000 & ENE de Ollantaytambo \\
Ríos Warán y & Patacancha & 4,050 & ENE de Ollantaytambo \\
Canchacancha & T'anchacancha & 3,700 & NNE de la ex hacienda Warán. \\
Río Lares & Pampacorral & 3,550 & ENE margen Izquierda del río Warán. \\
& Kiswarani & 3,400 & SSE de Lares, margen izquierda del río Maukao. \\
Río Trapiche & Tambowaylla & 3,600 & S de Lares, margen izquierda del río Maukao. \\
& Wakawasi & 3,400 & W de Lares, margen derecha del río Trapiche \\
& Kunkani & 3,800 & W de Lares, entre los ríos Pukamayu y Kurukumayu. \\
\hline \hline
\end{tabular}




\section{Metodología}

En campo, se midió la circunferencia basal, circunferencia distal y la longitud del fuste (MINAGRI, DGFFS \& PCM, OSINFOR, 2012). Para la determinación del volumen de leña en los bosques, se mensuraron árboles y arbustos con diámetro superior a $10 \mathrm{~cm}$ a una altura de $1.30 \mathrm{~m}$ siguiendo la metodología propuesta en el Manual base para la planificación y ejecución de inventarios forestales en bosques de producción permanente (RM $\underline{\mathrm{N}^{\circ} 0172-2012-\mathrm{AG}}$ ) y el Protocolo para la evaluación de individuos maderables (DGFFS-MINAGRI \& OSINFOR, 2012), definido como diámetro a la altura del pecho (DAP) utilizado para calcular el volumen del tronco de los árboles, con el objeto de obtener la productividad de leña en el bosque (FAO, 1983) y para la mensuración en los matorrales, la circunferencia basal mínima corresponde a tres cm o más.

El estudio se desarrolló durante el año 2007 en 12 comunidades campesinas, donde la unidad de análisis para la oferta de biomasa vegetal fue el metro cúbico $\left(\mathrm{m}^{3}\right)$, para la oferta de leña utilizada, la tonelada métrica (TM) y para consumo persona/día, el kilogramo.

Para recabar la información se aplicó un cuestionario estructurado de 25 preguntas con alternativas de respuesta. Se entrevistaron a 12 líderes comunales, 30 campesinos durante el recojo de leña y durante la acción de traslado y almacenamiento. Finalmente se interactuó con 20 amas de casa en la acción de preparar alimentos utilizando la leña colectada. Estos tres niveles de información, posibilitaron clasificar la selección y preferencia de la especie para ser clasificada en alta, media y baja. El análisis de la información se realizó utilizando estadística descriptiva, como frecuencias y-.

\section{promedios.}

Los nombres de las especies registradas fueron contrastados y consultados con la base de datos de Trópicos del Missouri Botanical Garden (www.tropicos.org) para la correcta redacción de los nombres científicos y los autores de las especies.

Las Zonas de Vida fueron determinadas mediante el Sistema Holdridge (Holdridge, 2000) y observaciones de campo, que es el utilizado en el país (ONERN,1975; AybarCamacho et al., 2017). Los datos bioclimáticos fueron obtenidos de las estaciones meteorológicas de Calca y Urubamba.

Para la obtención del volumen de madera, humedad y densidad se emplearon métodos experimentales en laboratorio. La humedad y capacidad calorífica de la madera fue calculada a través del secado al horno y evaporación en el laboratorio de Física de la Facultad de Ciencias de la UNSAAC.

El cálculo del consumo diario de leña de uso doméstico, se midió en kilogramos (kg), eligiendo los siguientes factores:

a. Información obtenida mediante encuestas sobre el consumo de combustible y las especies empleadas como leña y las mensuraciones efectuadas en campo.

b. Tiempo y mano de obra para obtener leña.

Para el cálculo de las unidades de medida, se midió el peso, en lugar del volumen. Durante las evaluaciones y mensuraciones en campo, se distinguieron cuatro tipos de unidades:

- La unidad normalizada, es la masa o volumen, $\left(\mathrm{kg} \mathrm{o} \mathrm{m}^{3}\right)$. Se utiliza cuando se compra, es la unidad en la que se basa el pago. Las medidas de peso se refieren a madera que- 
-no está seca; las medidas de volumen se refieren a un volumen apilado y no macizo. La conversión de estas unidades normalizadas a pesos equivalentes de madera secada al aire, se efectuó mediante mediciones del peso como del contenido de humedad.

- La unidad aproximada, es una medida no normalizada y definida aproximadamente, como una carga, tercio o “q'epe”. Se utiliza para la leña comprada y la recogida. Se utiliza un factor de conversión normalizado, basado en mediciones hechas en comunidades que utilizan la compra-venta; el varón recolecta una carga con un peso entre 48 y $55 \mathrm{~kg}$. y un tercio es el equivalente a 30 o 35 $\mathrm{kg}$; incluso ha de diferir si se trata del "q' epe" o tercio recolectado por la mujer $y$, una acémila puede cargar dos tercios de $35 \mathrm{~kg}$. ello depende del camino de herradura a recorrer, en caminos con menor pendiente, las acémilas portan hasta dos cargas.

- La unidad individual; cantidad de leña utilizada en un tiempo específico, como un día o una semana. El cálculo depende de qué especies contiene el tercio de leña colectado. Se midió el volumen y peso; se obtuvieron muestras para determinar la densidad y humedad.

- La unidad monetaria, es una medida indirecta de pago en dinero cuando se compra leña. La conversión a un peso equivalente requiere conocer tanto la unidad directa de compra que puede ser una unidad normalizada o aproximada, como el precio pagado. Al hacer leña, los campesinos utilizan dos horas para obtener una carga o "q'epe", esto es, el equivalente a $52 \mathrm{~kg}$. efectuando la conversión a unidad monetaria, implica un jornal diario de S/.20.00 (U.S.\$ 6.5), esto- -incluye el tiempo utilizado para trasladarse al bosque y el retorno, cuatro horas, usualmente en domingo.

Para la evaluación sobre la preferencia en el uso de las especies, cuando la preferencia fue superior al 70\% se la calificó como de alto uso; si la preferencia fue de $20 \%$ se consideró como de medio uso y, si la preferencia fue igual o inferior al 10\%, el uso de la leña se consideró como bajo.

\section{Resultados}

\section{Zonas de vida}

En el ámbito de estudio, se determinaron cuatro zonas de vida, que a continuación se describen brevemente.

\section{a. Monte Espinoso Subtropical -mte-S}

Gradación transicional hacia el monte espinoso pre montano; ocupa las partes bajas de las micro cuencas de Patacancha y Cancha cancha; entre Ollantaytambo y la comunidad Phallata, en el piso de valle; entre los 2,600 y 3,200 msnm. Los suelos están en pleno uso, con vegetación disturbada por la actividad agrícola y pecuaria. Las especies más representativas son: molle (Schinus molle); china molle (Schinus pearcei), P'ispita (Acalypha aronioides), tara (Caesalpinia spinosa), chinchircuma (Mutisia acuminata) y ch'eqche (Berberis boliviana); se observan plantaciones de Eucaliptus globulus y Alnus acuminata.

\section{b. Bosque Seco Montano Bajo Sub tropical-bs-MBS}

Subhúmeda, con árboles con epifitismo, ocupa el piso de valle y laderas de la micro cuenca del río Patacancha, corresponde a- 
ambos flancos del río Yuraqmayu. Presenta una vegetación mixta en asocies, característica de esta zona de vida, ubicada entre los 3,400 y 3,900 msnm. Hacia las riberas del ríoWarán, se observan: Alnus acuminata (aliso), Weinmania pentaphylla (wichullo), Hesperomeles lanuginosa (mayu manzana), Myrcine pellucida (chalanque), Myrica pubescens (laurel de puna) y hacia las laderas asocies de Duranta mandonii (mot'e mot'e); Hesperomeles heterophylla (quisca mayu manzana), Hesperomeles latifolia (lenle); Vallea stipularis (chiqllurmay); Citharexylum argutedentatum (kuruchu); Escallonia herrerae (chachacomo). En el bosque de Choqechaka, además se registra: Myrcianthes oreophila (unca), que tipifica este bosque; Tetraglochin cristatum (china canlli); Kageneckia lanceolata (lloque); Piper elongatum (moqo moqo) y junto al río, Alnus acuminata (aliso).

\section{c. Estepa Montano Subtropical. e- MS}

Constituye una transición hacia el Matorral Desértico, Subandino Subtropical, md-SaS. Altitudinalmente antecede a la Zona de Vida anterior e incluso se entremezclan constituyendo ecotonías; se ubica entre los 3,200 y 3,800 msnm. Especies más conspicuas: Baccharis carinata (monte cheqche); Berberis commutata (waca asta); Barnadesia horrida (llaulli); Barnadesia berberoides (jatun llaulli); Dasyphyllum leiocephalum (t'ankar llaulli); Duranta mandonii (mot'e tankar); Puya herrerae (achupalla).

\section{d. Paramo Húmedo Sub andino Subtropical.ph-SaS \\ Ubicada por encima de los 4,200 msnm. se observan rodales de Baccharis odorata (tayanka); Baccharis buxifolia (Chillca); Berberis boliviana (ch'eqche); Buddleja coriacea (qolle) y especies}

del género Polylepis.

\section{Especies más utilizadas en leña}

Se identificaron 56 especies utilizadas como leña; los resultados provienen del análisis de las entrevistas aplicadas a los actores sociales (líderes comunales, campesinos en el proceso de obtener, transportar y almacenar la leña y, amas de casa en la preparación de alimentos), las mismas que pertenecen a 22 familias, 36 géneros y 56 especies. De las especies identificadas, 46 son consideradas como de alto uso, cinco como de uso medio y otros cinco de uso bajo; significando la importancia de las especies en el bosque nativo de los altos andes en la oferta de leña.

El material energético de la biomasa vegetal, corresponde a vegetación arbustiva y arbórea de los bosques. Existe mayor diversidad en los bosques y bosquetes inferiores a 3,500 msnm constituyendo bosques mixtos, matorrales espinosos y asociaciones interespecíficas. En cambio, a altitudes superiores a los 3,500 m se observan rodales de Baccharis, Polylepis, Gynoxis, Weinmannia, Cytharexilum, Buddleja.

De las especies más utilizadas en el abastecimiento de leña, destacan por su abundancia los géneros Hesperomeles, Buddleja, Escallonia y Polylepis; sin embargo, considerando la biomasa que ofertan, las especies más importantes pertenecen a los géneros Alnus, Acalypha, Baccharis, Myrica, Berberis, Duranta, Barnadesia, Gynoxis, Hesperomeles, Myrcianthes, Citharexylum, Escallonia y Polylepis. 
Tabla 2. Especies utilizadas como leña en las comunidades campesinas de la cordillera del Vilcanota.

\begin{tabular}{|c|c|c|c|c|}
\hline Familia & Nombre científico & Nombre común & Hábito & $\begin{array}{c}\text { Nivel de uso como } \\
\text { combustible }\end{array}$ \\
\hline Betulaceae & Alnus acuminata Kunth & Aliso & Árbol & Alto \\
\hline Cunoniaceae & Weinmannia pentaphylla Ruiz \& Pav & Wichullo & Árbol & Alto \\
\hline Euphorbiaceae & Acalypha aronioides Pax \& K. Hoffm & P'ispita & Arbusto & Alto \\
\hline Euphorbiaceae & Sebastiana obtusifolia (H.B. K.) Pax \& K. Hoffm & Vinagrillo & Arbolillo & Alto \\
\hline Asteraceae & Aristeguietia discolor R.M king \& H. Rob & Vino vino & Arbusto & Bajo \\
\hline Asteraceae & Baccharis buxifolia (Lam.) Pers & Chillca & Arbusto & Alto \\
\hline Asteraceae & Baccharis latifolia (Ruiz \& Pav.) Pers & Mayu Chilca & Arbusto & Alto \\
\hline Asteraceae & Baccharis odorata Kunth & Tayanka & Arbusto & Alto \\
\hline Anacardiaceae & Schinus molle $L$ & Molle & Árbol & Alto \\
\hline Anacardiaceae & Schinus pearcei Engl & China molle & Arbolillo & Medio \\
\hline Flacourtaceae & Pineda incana Ruiz \& Pav & Olivero & Arbusto & Medio \\
\hline Myricaceae & Morellea pubescens (Humb. Bonpl ex Will d) Wilbur & Laurel de puna & Árbol & Alto \\
\hline Berberidaceae & Berberis carinata Lechl & Monte cheqche & Arbusto & Alto \\
\hline Berberidaceae & Berberis boliviana Lechl & Cheqche & Arbusto & Alto \\
\hline Berberidaceae & Berberis commutata Eichler & Waka asta & Arbolillo & Alto \\
\hline Asteraceae & Barnadesia horrida (Humb. Bonpl ex Willd) Wilbur & Llaulli & Arbusto & Alto \\
\hline Asteraceae & Bamadesia berberoides Sch. Bip. & Llaulli & Arbusto & Alto \\
\hline Asteraceae & Dasyphyllum leiocephalum (Wedd.) Cabrera & Táncar llaulli & Arbusto & Alto \\
\hline Verbenaceae & Duranta mandonii Moldenke & Mot'e mot'e & Arbolillo & Alto \\
\hline Asteraceae & Gynoxys longifolia We dd. & Q'oto kiswar & Arbolillo & Alto \\
\hline Asteraceae & Gynoxys callacallana Cuatrec & Qóto kiswar & Arbusto & Alto \\
\hline Asteraceae & Gynoxys nítida Muschl. & Tóqarway & Árbol & Alto \\
\hline Rosaceae & Hesperomeles heterophylla Hook & Kiska mayu manzana & Arbusto & Alto \\
\hline Rosaceae & Hesperomeles lanuginosa Ruiz \& Pav. ex Hook & Mayu manzana & Árbol & Alto \\
\hline Rosaceae & Hesperomeles latifolia (Kunth) M. Roem. & Lenle & Árbol & Alto \\
\hline Myrtaceae & Myrcianthes oreophilla (Diels) McVaugh & Unca & Árbol & Alto \\
\hline Araliaceae & Oreopanax ischnolobus Harms & Maqui maqui & Arbusto & Bajo \\
\hline Columelliaceae & Columellia obovata Ruiz \& Pav. & Huamanpinta & Arbusto & Alto \\
\hline Solanaceae & Saracha punctata Ruiz \& Pav. & Chiñuelas & Arbolillo & Alto \\
\hline Solanaceae & Cestrum conglomeratum Ruiz \& Pav. & Ñukao & Arbusto & Medio \\
\hline Solanaceae & Lycianthes lycioides (L.) Hassl. & Tánkar quiska & Arbusto & Alto \\
\hline Solanaceae & Dunalia spinosa (Meyen) Dammer & T'ankar & Arbusto & Alto \\
\hline Myrsinaceae & Myrsine pelucida (Ruiz \& Pav.) Spreng & Chalanque & Árbol & Medio \\
\hline Melastomataceae & Brachyotum naudinii Triana & Thire & Arbusto & Bajo \\
\hline Asteraceae & Mutisia acum inata Ruiz \& Pav & Chinchircuma & Arbusto & Medio \\
\hline Rosaceae & Margiricarpus cristatu s Britton & China Kanlli & Arbusto & Alto \\
\hline Bromeliaceae & Puya herrerae Harms & Achupalla & Roseta & Bajo \\
\hline Buddlejaceae & Buddleja coriácea J. Rémy & Qolle & Arbolillo & Alto \\
\hline Buddlejaceae & Buddleja incana Ruiz \& Pav. & Kiswar-kiswar & Árbol & Alto \\
\hline Elaeocarpaceae & Vallea stipularis L.f. & Chiqllurmay & Árbol & Alto \\
\hline Verbenaceae & Citharexylum argutedentatum Moldenke & Kuruchu & Árbol & Alto \\
\hline Caesalpinaceae & Senna birostris (Dombey ex Vogel) H.S. Irwin \& Barneby & Mutuy & Arbusto & Alto \\
\hline Caesalpineaceae & Senna multiglandulosa (Jacq.) H.S. Irwin \& Barneby & Asnaq mutuy & Arbolillo & Alto \\
\hline Caesalp iniaceae & Caesal pinia spinosa (Molina) Kuntze & Tara & Árbol & Alto \\
\hline Escalloniaceae & Escallonia herrerae Mattf. & Qeswa Chachacomo & Árbol & Alto \\
\hline Escalloniaceae & Escallonia resinosa (Ruiz \& Pav.) Pers. & Chachacomo & Árbol & Alto \\
\hline Escalloniaceae & Escallonia myrtilloides L.f. & T'asta & Árbol & Alto \\
\hline Rosaceae & Kageneckia lanceolata Ruiz \& Pav & Lloque & Arbolillo & Alto \\
\hline Piperaceae & Piper elongatum Vahl & Moq'o moq'o & Arbusto & Bajo \\
\hline Rosaceae & Polylepis racemosa Ruiz \& Pav & Queuña & Árbol & Alto \\
\hline Rosaceae & Polylepis incana Kunth & Queuña & Árbol & Alto \\
\hline Rosaceae & Polylepis pauta Hieron & Queuña & Árbol & Alto \\
\hline Rosaceae & Polylepis subsericans J. F. Macbr & Queuña & Árbol & Alto \\
\hline Rosaceae & Polylepis sericea Wedd. & Queuña & Árbol & Alto \\
\hline Rosaceae & Polylepis pepei B.B. Simpson & Queuña & Arbolillo & Alto \\
\hline Rosaceae & Polylepis microphylla (Wedd.) Bitter & Queuña & Arbusto & Alto \\
\hline
\end{tabular}


Parámetros físico químicos de leña utilizada

\section{A. Contenido de humedad}

El método tradicional del secado en las comunidades, consiste en la exposición del producto húmedo al sol y al aire libre. Las- -características de la humedad ambiental, $60 \%$ promedio anual de la zona andina (Toivonen \& Kessler, 2006) son propicias; pero, es un proceso lento, hasta alcanzar la humedad de equilibrio. El tiempo de secado varía de una especie a otra y de las condiciones meteorológicas.

Tabla 3. Variables físicas de humedad y densidad en muestras de madera y leña nativas para especies en la micro cuenca del río Málaga, Comunidad deT'astayoq.

\begin{tabular}{l|c|c|c|c|}
\hline \multicolumn{1}{c|}{ Especies Nativas } & mh (g) & ms (g) & Hh (\%) & Hs (\%) \\
\hline Queuña (Polylepis racemosa) & 97 & 64 & 34.02 & 51.56 \\
Chiñuelas (Saracha punctata) & 142 & 119 & 16.20 & 19.33 \\
T'asta (Escallonia myrtilloides) & 127 & 93 & 26.77 & 36.56 \\
Tayanca (Baccharis odorata) & 61 & 44 & 27.87 & 38.64 \\
Lenle (Hesperomeles latifolia) & 77 & 63 & 18.18 & 22.22 \\
Kurucho (Citharexylum argutedentatum) & 99 & 87 & 12.12 & 13.79 \\
Mot'e mot'e (Duranta mandonii) & 111 & 98 & 11.71 & 13.27 \\
Aliso (Alnus acuminata) & 71 & 62 & 12.68 & 14.52 \\
Eucalipto (Eucaliptus globulus) & 142 & 127 & 10.56 & 11.81 \\
\hline
\end{tabular}

Datos en base a resultados del análisis en laboratorio de Física. UNSAAC. $\mathrm{M}_{\mathrm{h}}$ : Masa con contenido de humedad; $\mathrm{m}_{\mathrm{s}}$ : Masa seca ( $\sin$ humedad), $\mathrm{H}_{\mathrm{h}}$ : Contenido de humedad en húmedo, $\mathrm{H}_{\mathrm{s}}$ : Contenido de humedad en seco.

\section{B. Poder calórico de las especies nativas más importantes}

El análisis químico especifica que el eucalipto posee seis veces más ceniza (A) que el kuruchu (Citharexylum argutedentatum), ocho veces más que el chachacomo (Escallonia resinosa) y 5.8 veces más que la t'asta (Escallonia myrtilloides). El eucalipto posee $4.3 \%$ de residuo sólido luego de la combustión, en las especies nativas, los valores fluctúan por debajo de la unidad. El kurucho posee $0.73 \%$, significando que, de la masa total de leña, menos del $1 \%$ es convertido en ceniza y gran parte se convierte en brasa útil. En el caso de la bosta, un alto nivel de porcentaje de la biomasa se convierte en ceniza. 
Tabla 4. Poder calorífico de especies nativas utilizadas como leña

\begin{tabular}{l|cccccccccc|c|c|}
\hline \multirow{2}{*}{$\begin{array}{l}\text { ESPECIES } \\
\text { NATIVAS }\end{array}$} & \multicolumn{7}{|c|}{ ANALISIS QUIMICO DE LAS } & \multicolumn{2}{c|}{ MUESTRA S } & \multirow{2}{*}{$\begin{array}{c}\text { PCI } \\
\text { (NJ) }\end{array}$} & $\begin{array}{c}\text { PCI } \\
\text { (Kcal/kg) }\end{array}$ \\
\hline Eucalipto & 0.15 & 0.06 & 39.3 & 52.4 & 6 & 12.33 & 4.3 & 12654.5759 & 3023.0712 \\
K'urucho & 0.25 & 0.02 & 39.1 & 52 & 5.98 & 21.5 & 0.73 & 12564.1101 & 3001.4596 \\
Mote-Mote & 0.24 & 0.07 & 38.52 & 51.23 & 5.98 & 16.4 & 0.57 & 12518.5715 & 2990.5809 \\
T'asta & 0.26 & 0.09 & 38.84 & 51.66 & 5.94 & 18.5 & 0.74 & 12503.0603 & 2986.8754 \\
Aliso & 0.27 & 0.05 & 38.6 & 51.34 & 5.9 & 12.2 & 0.61 & 12455.3684 & 2975.4822 \\
Chachacomo & 0.28 & 0.05 & 38.34 & 46.82 & 5.38 & 19.86 & 0.52 & 12375.4444 & 2956.389 \\
Lenle & 0.26 & 0.05 & 38.4 & 51.07 & 5.87 & 20.8 & 0.88 & 12340.1749 & 2947.9634 \\
Qolle & 0.24 & 0.02 & 37.6 & 45.5 & 5.4 & 13.5 & 0.65 & 12383.2486 & 2958.2534 \\
Lloque & 0.29 & 0.04 & 38.2 & 50.81 & 5.84 & 16.2 & 0.68 & 12301.7347 & 2938.7804 \\
Queuña & 0.5 & 0.09 & 37.97 & 50.6 & 5.8 & 23.52 & 0.83 & 12167.4024 & 2906.6895 \\
Tayanca & 0.3 & 0.06 & 36.7 & 48.81 & 5.61 & 14 & 0.89 & 11829.9787 & 2826.0819 \\
Chiqllurmay & 0.26 & 0.04 & 35.82 & 47.64 & 5.48 & 16.2 & 0.71 & 11534.8050 & 2755.5674 \\
Bosta & 2.52 & 0.74 & 32.88 & 43.73 & 5.03 & 24.9 & 11.18 & 10592.9504 & 2530.5663 \\
\hline
\end{tabular}

C: Carbono, H: Hidrógeno, O: Oxígeno, S: Azufre, W: Agua, A: Cenizas

La siguiente tabla evidencia los valores -estudios efectuados por Géhu (1981) y, transformados en Kcal/kg y en KJ/kg para las Pedrotti et al. (1992).

especies nativas, tomando como base los-

Tabla 5. Poder calorífico de las especies nativas al 25\% de humedad

\begin{tabular}{|c|c|c|c|c|c|}
\hline Especie & Nombre local & $\begin{array}{l}\text { Densidad } \\
\mathrm{kg} / \mathrm{m}^{3}\end{array}$ & $\begin{array}{l}\text { Energía } \\
\left(\mathrm{Gcal} / \mathrm{m}^{3}\right) \\
\text { al } 25 \% \text { de } \\
\text { humedad }\end{array}$ & 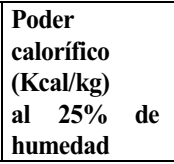 & $\begin{array}{l}\text { Poder calorífico } \\
(\mathrm{KJ} / \mathrm{kg}) \\
\text { al } 25 \% \text { de } \\
\text { humedad }\end{array}$ \\
\hline Escallonia re sinosa & Chachacomo & 810 & 1.68 & 2074.07 & 8682.07 \\
\hline Polypelis incana & Queña & 630 & 1.44 & 2285.71 & 9568.00 \\
\hline Escallonia myrtilloides & Tasta & 710 & 1.22 & 1718.31 & 7192.85 \\
\hline Eucaliptus globulus & Eucalipto & 720 & 1.18 & 1638.89 & 6860.39 \\
\hline Myrcianthes oreophilla & Unca & 790 & 1.18 & 1493.67 & 6252.51 \\
\hline $\begin{array}{l}\text { Citharexylum } \\
\text { xrgutedentatum }\end{array}$ & K'uruchu & 820 & 1.08 & 1317.07 & 5513.27 \\
\hline Kageneckia lanceotata & Lloque & $\mathrm{s} / \mathrm{i}$ & 1.08 & $\mathbf{s} / \mathbf{i}$ & $\mathbf{s} / \mathbf{i}$ \\
\hline Alnus acuminata & Aliso & 470 & 0.99 & 2106.38 & 8817.32 \\
\hline Vallea stipularis & Chiqllurmay & 610 & 0.92 & 1508.20 & 6313.31 \\
\hline Buddleja coriacea & Qolle & $\mathrm{s} / \mathrm{i}$ & 0.91 & $\mathbf{s} / \mathbf{i}$ & $\mathbf{s} / \mathbf{i}$ \\
\hline Hesperomeles latifolia & Lenle & 740 & 0.90 & 1216.22 & 5091.08 \\
\hline Duranta mandonii & Mot'e & 530 & 0.90 & 1698.11 & 7108.30 \\
\hline
\end{tabular}

Datos en base a información de Géhu, J.M-Pedrotti, y convertida a unidades de energía. 
C. Consumo de leña en las comunidades altoantinas

\section{Obtención de leña}

La recolección de leña, ramas, chamarasca y troncos, en las comunidades de Canchacancha y Phallata, no requiere una dedicación especial de tiempo. A excepción de la leña de "raja" que es tarea de varones a fin de proveer leña para la semana; la mujer y los jóvenes pastores recolectan leña que encuentran en su recorrido junto al ganado y llevan al final de la jornada a casa, constituye la provisión diaria, es la leña más seca y con buen rendimiento.

En las comunidades por encima de los 3,500 msnm la escasez de leña, y el trabajo que cuesta obtenerla, induce a no desperdiciarla. Existe una división de trabajo en la obtención de leña, el varón puede obtener hasta una carga o "q'epe" de 4 arrobas; la mujer tiene obligación de obtener una carga o "q'epe" de una arroba y leña de chamarasca en esfuerzos por salida.

\section{Unidades de Medida}

Como se observa en la Tabla 6, las unidades de medida locales difieren de una comunidad a otra, en función a distancias, al tipo de leña, al acceso al bosque y si se trata de un hombre o de- -mujeres y niños como colectores de leña; por lo tanto, a efectos de estandarizar las medidas de peso, se ha considerado la carga como equivalente a $52 \mathrm{~kg}$. Esta unidad de medida, respecto del tiempo de su recolección y/o preparación, también difiere, dependiendo si se trata de colectar en bosques de Polylepis, en bosques mixtos o en matorrales espinosos. Es el caso del bosque mixto de Phallata (Choquechaka), un hombre tarda 2 horas y 30 minutos para colectar una carga; en cambio para colectar el mismo peso en los bosques de Canchacancha, se demora una hora y 15 minutos; esto es debido a la oferta de leña en el bosque. Análogamente en los matorrales espinosos de Kiswarani, Pampacorral, Willoq, Tambowaylla, para obtener una carga del mismo peso $(52 \mathrm{~kg})$, demoran más de tres horas; significando ello un mayor esfuerzo para obtener un mismo peso de leña, debido a la escasez de la oferta. De otro lado, cuando los comuneros obtienen leña de los bosques de Polylepis, sólo el tiempo para colectar una carga de $52 \mathrm{~kg}$. es de 3 horas con 30 minutos; ello en razón a que la leña de Polylepis exige mayor dedicación, no sólo por la colecta, sino el trozar, cortar y astillar lleva un mayor tiempo.

Tabla 6. Unidades de medida local para la colecta de leña y sus equivalentes en kg.

\begin{tabular}{|l|l|l|l|}
\hline Comunidad & $\begin{array}{l}\text { Unidad de } \\
\text { medida } \\
\text { local }\end{array}$ & $\begin{array}{l}\text { Peso } \\
\text { equivalente } \\
\text { (kg) }\end{array}$ & \multicolumn{1}{c|}{ Observaciones } \\
\hline Phallata & Carga & 52 & $\begin{array}{l}\text { La carga de un varón equivale a cuatro arrobas (52 } \\
\text { kg) y para mujeres a una arroba. Una acémila pueda } \\
\text { portar dos cargas, c ada uno de } 45 \mathrm{~kg} .\end{array}$ \\
$\begin{array}{l}\text { Rumira - } \\
\text { Sondor }\end{array}$ & Carga & $40-45$ & $\begin{array}{l}\text { La llama puede portar una carga de una arroba o } 15 \\
\text { kg. y el caballo dos cargas, cada uno de 30 kg. }\end{array}$ \\
\hline
\end{tabular}




\begin{tabular}{|c|c|c|c|}
\hline Willoq & $\begin{array}{l}\text { Carga, } \\
\text { tercio }\end{array}$ & $\begin{array}{l}40 \\
15\end{array}$ & $\begin{array}{l}\text { La carga es sólo empleada para la leña de queuña o de } \\
\text { eucalipto. El varón traslada una carga y el caballo dos } \\
\text { cargas cada uno de unos } 25 \mathrm{~kg} \text {. esta diferencia de peso } \\
\text { se debe, a la pendiente y al estado del camino. El } \\
\text { bosque de donde obtienen leña es Queuñaqocha. El } \\
\text { tercio es la unidad de medida para la colecta de leña } \\
\text { procedente del matorral espinoso. }\end{array}$ \\
\hline Canchacanccha & $\begin{array}{l}\text { Q'epe, } \\
\text { tercio }\end{array}$ & $\begin{array}{l}50-55 \\
8-10\end{array}$ & $\begin{array}{l}\text { El q'epe equivale a } 4 \text { arrobas o } 52 \mathrm{~kg} \text {. Esta } \\
\text { comunidad, colecta leña para vender en } \\
\text { Ollantaytambo o para hacer carbón y el q'epe cuesta } \\
\text { aproximadamente S/. 20.00. Los adolescentes y } \\
\text { mujeres colectan leña en forma diaria y traen al final } \\
\text { de la jornada un tercio de leña bastante seca. }\end{array}$ \\
\hline Pampacorral & $\begin{array}{l}\text { Tercio } \\
\text { Carga }\end{array}$ & $\begin{array}{l}30-35 \\
50-55\end{array}$ & $\begin{array}{l}\text { Un tercio equivale a } 32 \mathrm{~kg} \text {. y es para el varón; para las } \\
\text { mujeres y niños el tercio equivale a } 15 \mathrm{~kg} \text {. Es utilizada } \\
\text { para leña del matorral espinoso; para la leña de } \\
\text { queuña, la unidad de medida es la carga. }\end{array}$ \\
\hline Kiswarani & $\begin{array}{l}\text { Carga } \\
\text { tercio }\end{array}$ & $\begin{array}{l}34 \\
15-20\end{array}$ & $\begin{array}{l}\text { La carga equivale a tres arrobas y debido a la escasez } \\
\text { de leña lo hacen en un día de trabajo. } \\
\text { El tercio equivale a media carga. }\end{array}$ \\
\hline Wakawasi & Q'epe & 23 & $\begin{array}{l}\text { Esta unidad fluctúa, depende de dónde y que leña } \\
\text { obtienen; si la leña proviene del bosque de Polylepis, } \\
\text { entonces el q'epe equivale a dos arrobas ( } 23 \mathrm{~kg}) \text {, el } \\
\text { caballo carga dos q'epes ( } 46 \mathrm{~kg} \text {.) y el hombre uno; } \\
\text { debido a la lejanía del bosque. Si la leña proviene del } \\
\text { matorral espinoso, el hombre porta el peso de dos } \\
\text { q'epes ( } 45 \text { a } 50 \mathrm{~kg} \text {.) }\end{array}$ \\
\hline
\end{tabular}

\section{Uso actual y requerimientos comunales}

En las comunidades altas, se estima entre 0.45 y $1.2 \mathrm{~m} 3$ las necesidades de leña por habitante/año; siendo la disponibilidad entre 0.2 y $0.4 \mathrm{~m} 3$; existiendo un déficit anual de 0.8 $\mathrm{m} 3$. En las comunidades de la micro cuenca del río Warán, la situación es mejor; la oferta de leña en los bosques naturales es buena; se estima en $1 \mathrm{~m} 3$ el consumo anual por habitante, del cual el 80\% es satisfecho con leña de bosques naturales, el resto proviene del eucalipto.

Tabla 7. Consumo de leña per cápita en las comunidades altoandinas.

\begin{tabular}{|c|c|c|c|c|}
\hline $\begin{array}{l}\text { Comuni } \\
\text { dad }\end{array}$ & $\begin{array}{l}\text { Leña nativa } \\
\text { (kg./persona/ } \\
\text { día) }\end{array}$ & $\begin{array}{l}\text { Bosta } \\
\text { (kg) }\end{array}$ & $\begin{array}{l}\text { Eucalipto } \\
\text { (kg) }\end{array}$ & Observaciones \\
\hline Phallata & 1.30 & 0.12 & 0.60 & $\begin{array}{l}\text { Calculada en } \mathrm{f} \text { unción a } 06 \text { miembros/familia; y una } \\
\text { carga }(52 \mathrm{~kg} \text { ) de leña nativa es suficiente para seis días. } \\
\text { Es la comunidad con menor consumo de bosta y buen } \\
\text { consumo de leña nativa, poseen un bosque excelente } \\
\text { con oferta de leña. }\end{array}$ \\
\hline
\end{tabular}




$\begin{array}{lccc}\begin{array}{l}\text { Cancha - } \\ \text { cancha }\end{array} & 1.44 & 0.14 & 0.00 \\ & & & \\ & & & \\ \begin{array}{l}\text { Pampa } \\ \text { corral }\end{array} & 1.07 & 0.27 & 0.7 \\ \begin{array}{l}\text { Kiswara } \\ \text { ni }\end{array} & 1.13 & 0.30 & 0.67 \\ \begin{array}{l}\text { Tambo - } \\ \text { waylla }\end{array} & 1.24 & 0.143 & 0.29 \\ \begin{array}{l}\text { Willoq } \\ \text { 1.06 }\end{array} & 0.25 & 0.20 \\ \begin{array}{l}\text { Rumira - } \\ \text { Sondor y } \\ \text { Pata } \\ \text { cancha }\end{array} & 0.82 & 0.31 & 0.20 \\ & & & 0.25 \\ \end{array}$

$\begin{array}{llll}\text { Kunkani } & 0.92 & 0.31 & 0.25\end{array}$

$\begin{array}{llll}\text { Wakawa } & 0.93 & 0.36 & 0.36 \\ \text { si }\end{array}$

\begin{tabular}{llll} 
PRO & 1.10 & 0.245 & 0.409 \\
ME- & & & \\
DIO & & & \\
\hline
\end{tabular}

Las comunidades ubicadas por debajo de los 3,500 msnm y que poseen bosques naturales de especies nativas, son las que mayor leña de estas especies consumen, es el caso de Phallata y Canchacancha; en cambio las comunidades ubicadas por encima de los 3,500 msnm consumen menos combustible leñoso, es el caso de Patacancha, Rumira-Sondor, Kunkani y Wakawasi; es de advertir también que las
Calculada en base a que una carga $(52 \mathrm{~kg}$.) suficiente para seis días en una familia de seis personas. Es la comunidad con más alto consumo de leña nativa, por tener bosques en más de 200 ha. No requieren al eucalipto y utilizan la bosta como "mec ha" para iniciar el fuego. La oferta de leña es suficiente.

Calculada para una familia de seis personas y la carga (32 kg) es suficiente para cinco días.

Calculado en función a que una carga $(34 \mathrm{~kg}$.) de leña nativa es su ficiente para cinco días de uso, acompañado por nueve $\mathrm{kg}$. de bosta y $20 \mathrm{~kg}$. de eucalipto, consumido en una familia de seis miembros.

Calculado en base a seis miembros/familia y el uso de $52 \mathrm{~kg}$. de leña nativa, seis $\mathrm{kg}$. de bos ta y $15 \mathrm{~kg}$. de eucalipto, utilizado para siete días de consumo.

Determinado en función a una familia de siete miembros y una carga de leña se consume en siete días. La comunidad utiliza en ese mismo tiempo $12 \mathrm{~kg}$. de bosta (vacuno y d e llama) y $10 \mathrm{~kg}$. de leña de eucalipto

Estas comunidades en promedio tienen siete miembros/familia y consumen a la semana $40 \mathrm{~kg}$ de leña nativa, $15 \mathrm{~kg}$. de bosta (vacuno y llama) y $10 \mathrm{~kg}$. de eucalipto. Además emplean un suelo turboso obtenido en bloques o "champas" de los humedales por encima de los 4,500 msnm.

Determinado en base a que la familia promedio tiene siete miembros y consumen $45 \mathrm{~kg}$. de leña nativa; $15 \mathrm{~kg}$. de bosta (vacuno y llama) y $12 \mathrm{~kg}$. de leña de eucalipto para un período de siete días.

Una familia promedio tiene siete mie mbros y $52 \mathrm{~kg}$. de leña nativa es utilizada hasta ocho días; la bosta (llama, alpaca, vacuno e incluso caballo) colectada para o cho días es de $20 \mathrm{~kg}$. y complementada con $20 \mathrm{~kg}$. de eucalipto.

El poblador de las comunidades andinas consume, en promedio, $1.754 \mathrm{~kg}$. de combustible al día. 
Tabla 8. Distribución porcentual del consumo de combustible en las comunidades andinas. $\mathrm{kg} /$ persona/día.

\begin{tabular}{l|l|l|l|l|l|l|l|}
\hline $\begin{array}{l}\text { Comunidad } \\
\text { campesina }\end{array}$ & $\begin{array}{l}\text { kg. Leña } \\
\text { nativa }\end{array}$ & $\%$ & $\begin{array}{l}\text { kg. } \\
\text { Bosta }\end{array}$ & $\%$ & $\begin{array}{l}\text { kg leña } \\
\text { eucalipto }\end{array}$ & $\%$ & $\begin{array}{l}\text { Total combustible } \\
\text { Utilizado } \\
\text { kg/persona/día }\end{array}$ \\
\hline Phallata & 1.30 & 64.36 & 0.12 & 5.94 & 0.60 & 29.7 & 2.02 \\
Cancha & 1.44 & 91.14 & 0.14 & 8.86 & 0.00 & 0.00 & 1.58 \\
Pampacorral & 1.07 & 52.45 & 0.27 & 13.24 & 0.7 & 34.31 & 2.04 \\
Kiswarani & 1.13 & 53.81 & 0.30 & 14.29 & 0.67 & 31.90 & 2.10 \\
Tambowaylla & 1.24 & 74.25 & 0.143 & 8.56 & 0.29 & 17.37 & 1.67 \\
Willoq & 1.06 & 70.2 & 0.25 & 16.56 & 0.20 & 13.25 & 1.51 \\
Rumira - & 0.82 & 51.19 & 0.31 & 19.62 & 0.20 & 12.66 & 1.58 (consumen además \\
Sondor, & & & & & & & terrones de turba en \\
Patacancha & & & & & & & $0.25 \mathrm{~kg} /$ persona/día) \\
Kunkani & 0.92 & 62.16 & 0.31 & 20.95 & 0.25 & 16.90 & 1.48 \\
Wakawasi & 0.93 & 56.36 & 0.36 & 21.82 & 0.36 & 21.82 & 1.65 \\
Promedio & $\mathbf{1 . 1 0}$ & $\mathbf{6 2 . 7 1}$ & $\mathbf{0 . 2 4 5}$ & $\mathbf{1 3 . 9 7}$ & $\mathbf{0 . 4 0 9}$ & $\mathbf{2 3 . 3 2}$ & $\mathbf{1 . 7 5 4}$ combustible/persona/ \\
& & & & & & día \\
\hline
\end{tabular}

Datos en base a resultado de mesuraciones directas y encuestas.

La tabla 8 evidencia que más del 60\% del combustible utilizado en las comunidades proviene de los bosques nativos; en algunas comunidades como en Phallata y Cancha cancha, la leña proviene de bosques mixtos y matorrales espinosos, en cambio en comunidades como Pampacorral, Willoq, Wakawasi y Patacancha, mayormente provienen de bosques de Polylepis.

\section{Discusiones}

Conservation International/Smithsonian Institution (2001), reporta que uno de los puntos críticos de biodiversidad más críticos (hotspot) son los ecosistemas de montaña, sus bosques, cuencas hidrográficas, biodiversidad y cultura humana, merecen un gran esfuerzo para conservarlo; las observaciones de campo, nos permiten colegir que también los ecosistemas de la Cordillera del Vilcanota, son singulares y también requieren acciones de conservación como la reforestación con- -especies nativas.

Géhu (1991) y Pedrotti et al. (1992) demuestran que existe una correlación directa entre la humedad y, la densidad de la madera con el poder calorífico de ésta; en efecto, los estudios de campo y laboratorio, demuestran una correlación alta.

Los trabajos de Bastos de Andrade et al (1989), evidencian que a menor humedad de la madera debe existir una mayor combustión con desprendimiento de mayor energía; los estudios de laboratorio, ratifican estos resultados.

Toivonen y Kessler, (2006), sostienen que los bosques de Polylepis representan la vegetación natural de una gran parte de los Andes centrales a altitudes entre 3, 500 y 4,400 msnm, nuestros estudios en la cordillera del Vilcanota verifican que los bosques monoespecíficos de Polylepis llegan a altitudes superiores de 4,600 msnm. 
Coincidimos con los resultados de Huaranca et al. (2006) y Armenteras et al., (2003) que sostienen que la fragmentación de los Bosques Andinos es un fenómeno cada vez más frecuente que altera la diversidad de los organismos que los habitan, influyendo en la provisión de los servicios y bienes del bosque.

Según FAO y PNUMA, 2020; los bosques se encuentran sujetos a numerosas perturbaciones como incendios forestales y fenómenos meteorológicos que afectan los servicios ecosistémicos; los trabajos de campo en las 12 comunidades altoandinas estudiadas, nos permiten puntualizar que es la ganadería y la extracción de leña las acciones más perturbadoras.

De conformidad a lo indicado por Mejía (2011), el 58\% de las familias utilizan leña y gas propano, el 17\% utiliza sólo leña; en cambio nuestro trabajo demuestra que el 100\% de las familias emplean leña proveniente de bosques nativos y bosta de ganado. Indica además que todas las especies tienen similar poder calorífico; en cambio nuestro trabajo demuestra que existe sustancial diferencia entre las especies utilizadas como combustible.

Los estudios de Córdova (2012), señalan que la oferta de leña en las comunidades del bajo Urubamba es alta y no requieren almacenar, excepto para actividades comunales; en cambio, en las comunidades altoandinas, se verificó que las familias almacenan la leña para un periodo mayor a una semana; igualmente, el mismo estudio concluye que no es un problema significativo el abastecimiento de leña; en cambio, en las comunidades altoandinas, la oferta de leña proveniente de bosques nativos va disminuyendo, especialmente por encima de los $3500 \mathrm{msnm}$.

\section{Conclusiones}

Las especies con mayor poder calorífico, pocos residuos de ceniza y poca producción de humo son: chachacomo, queuña, t'asta, unca, k'uruchu, lloque, lenle, aliso, entre la vegetación arbórea y, waka asta, llaulli, entre los arbustos. El chachacomo, t'asta, unca, qolle, aliso, chiqllurmay y queuña son maderas apreciadas para la construcción de infraestructuras y aperos.

Las comunidades campesinas de la cordillera del Vilcanota consumen 1.754 $\mathrm{kg} /$ persona/día de leña; más del $60 \%$ proviene debosques nativos.

Especies nativas más utilizadas como leña son: chachacomo (Escallonia resinosa), queuña (Polylepis spp.), Tayanka (Baccharis odorata), T'asta (Escallonia myrtilloides), Lenle (Hesperomeles latifolia), aliso (Alnus acuminata), Unca (Myrcianthes oreophilla), Chiqllurmay (Vallea stipularis), K'uruchu (Citharexylum argutedentatum), q'otokiswar (Gynoxys longifolia), lloque (Kageneckia lanceolata).

La oferta de leña para comunidades por debajo de 3,500 msnm es mayor y proveniente de bosques mixtos y matorrales. En las comunidades sobre 3,500 msnm, la vegetación es escasa, emplean mayor bosta de ganado vacuno, ovino y de camélidos sudamericanos; la leña proviene del bosque de Polylepis.

Todas las comunidades emplean bosta de ganado, las comunidades altas prefieren la bosta de la llama (takia).

Las especies nativas en estado fresco poseen un poder calorífico que fluctúa entre $11,500 \mathrm{a}$ $12,500 \mathrm{KJ} / \mathrm{kg}$ y, a un 25\% de humedad, las especies Escallonia resinosa, Escallonia myrtilloides, Myrcianthes oreophilla; Citharexylum argutedentatum, Vallea stipularis, Hesperomeles latifolia, Duranta mandonii; evidencian un- 
poder calorífico superior a $8,000 \mathrm{KJ} / \mathrm{kg}$. Indicando que éstas especies nativas, deberían ser de prioridad en los proyectos de reforestación.

La leña proveniente de bosques nativos tiene una función preponderante en el aprovisionamiento energético de las poblaciones rurales y de los grupos más pobres de los centros urbanos. La leña ocupa un lugar especial, debido a la importancia del consumo doméstico de energía, al que la leña se destina principalmente, y debido al hecho de que constituye un servicio ecosistémico de los bosques naturales altoandinos.

Las especies nativas estudiadas y, particularmente el K'urucho (Citharexylum argutedentatum), una de las especies más apreciadas por el poder calorífico que posee su leña. Debe ser utilizada en la reforestación de los altos andes.

\section{Agradecimientos}

A Ecosistemas Andinos-ECOAN, por posibilitar la ejecución del estudio, al colega AlfredoTupayachi Herrera por la identificación de las especies botánicas, al Ing. Santos Mera Terrones, especialista en Geomática, por contribuir con el mapa de ubicación.

\section{Referencias bibliográficas}

Ansión, J. y Van Dam, C. (1986). El árbol y el bosque

en la sociedad andina. Proyecto FAO/Holanda/INFOR. Talleres Gráficos Art. Lantrec. Lima. Perú. 119 p.

Armenteras, D., Gast, F., Villareal, H. (2003). Andean forest fragmentation and the representativeness of protected natural areas in the Eastern Andes, Colombia. Biological Conservation 113:245-256.
Ataroff, M., Rada, F. (2000). Deforestation impact on water dynamics in a Venezuelan Andean cloud forest. AMBIO: A Journal of the Human Environment 29. 440-444p.

Aybar-Camacho, C.; Lavado-Casimiro, W.; Sabino, E.; Ramírez, S.; Huerta, J. \& FelipeObando, O. (2017). Atlas de zonas de vida del Perú -Guía Explicativa. Servicio Nacional de Meteorología e Hidrología del Perú (SENAMHI). Dirección de Hidrología. Lima. 27p.

Aubad, J.; Aragón, P.; Olalla-Tárraga, M. \& Rodríguez, M.A. (2008). Illegal logging, landscape structure and the variation of tree species richness across North Andean forest remnants. Forest Ecology and Management 255:1892-1899.

Bastos de Andrade, E. - Sasseron, José Luis Oliveira Filho, Delly. (1989). Principios sobre combustibles, combustión y hornos-fogones. CENTREINAR, Brasil.

Bermejo, J. Passeti, F. (1985). El árbol en apoyo de la Agricultura. Proyecto FAO/Holanda/INFOR. Lima. Documento de trabajo n ${ }^{\circ} 4.44$ pp.

Bubb, P., May, I., Miles, L., Sayer, J. (2004). Cloud ForestAgenda. UNEP-WCMC. Cambridge, UK.

Conservation International/Smithsonian Institution. (2001). Biological and Social Assessments of the Cordillera delVilcabamba, Peru. RAP. Conservation International. Washington D.C. $296 \mathrm{p}$.

Córdova, P. (2012). Estudio del consumo de leña en dos comunidades nativas de la cuenca del rio Bajo Urubamba. UNA La Molina. Lima. 73 p.

Desarrollo Forestal Participativo. (1992). El Verdor de los Andes. Editora Luz de América. Quito, Ecuador. 217 pp.

De Rutté Corzo, Jano. (2014). Composición y diversidad arbórea de un área de bosque montano en la concesión para la Conservación Puyu Sacha, Chanchamayo, Junín. Tesis, Ing. Forestal. UNALM. Lima. 136 p. 
ECOBONA/INTERCOOPERATION. (2009). Incidencia política para la gestión social de ecosistemas forestales Andinos. Análisis y propuesta para el Perú. Serie Investigación y Sistematización $03.107 \mathrm{pp}$.

Etter, A.;Villa, A. (2000). Andean forests and farming systems in part of the Eastern Cordillera (Colombia). Mountain Research and Development 20(3): 236-245.

FAO. (1981). Mapa de la situación en materia de leña en los países en desarrollo. Roma.

FAO. (1983). Disponibilidad de leña en los países en desarrollo. Estudio FAO Montes 42. Roma Italia. D i s p o n i b l e e $n$ : www.fao.org/3/x5329s/x5329s02.htm

FAO y PNUMA. (2020). El estado de los bosques del mundo 2020. Los bosques, la biodiversidad y las p e r s o n a s. $\mathrm{R}$ o $\mathrm{m}$ a . https://doi.org/10.4060/ca8642es.

Géhu, J.M. \& S. Rivas-Martínez (1981): Notions fondamentales de phytosociologie. In: Dierschke, H. (ed.) Syntaxonomie. Ber. Intern. Symposium IV-V: 5-53. Ed. Cramer,Vaduz.

Géhu, J.M. (1991). L'analyse phytosociologique et géosymphytosociologique de l'espace. Théorie et méthodologie. Coll. Phytosoc. 17: 11-46.

Geist, H. J., Lambin F. (2001). What drives tropical deforestation? A meta-analysis of proximate and underlying causes of deforestation based on subnational case study evidence. Louvain-la-Neuve, Belgium, University of Louvain. 110 p. LUCC Report Series $\mathrm{N}^{\circ} 4$.

Gentry, A. H. (1995). Patterns of diversity and floristic composition in neotropical montane forests. En: Churchill, S.P., Balslev, H., Forero, E., Luteyn, J.L. (eds.). Neotropical montane forest biodiversity and conservation symposium (1993, Bronx, N.Y, USA). Biodiversity and conservation of neotropical montane forests: Proceedings, pp. 103-126. New York Botanical Garden, NewYork, USA.
Gil Mora, J.E. (2000). Deforestación y Crisis de Leña. Revista Oropesa $\mathrm{N}^{\circ}$ XXV. Cusco, Perú. 22 p.

Gil Mora, J.E. (2002). Deforestación: Principales causas y efectos ambientales. Revista Oropesa $\mathrm{N}^{\circ}$ XLIII. Cusco, Perú. 23 p.

Holdridge, Leslie R. (2000). Ecología basada en zonas de vida. Quinta reimpresión. Instituto Interamericano de Cooperación para la Agricultura. San José, Costa Rica. 159 p.

Huaranca, J.C.; Ruiz, O.; Fernández, M. (2006). Folivoría en fragmentos de bosque de Polylepis besseri en Sacha Loma, Cochabamba, Bolivia. II Congreso de Ecología y Conservación de Bosques de Polylepis - Cusco, Perú.

INRENA (1995). Mapa ecológico del Perú. Guía explicativa. Lima. $271 \mathrm{p}$.

Jadan S. (1989). Selección de especies forestales, región Andina del Ecuador. Dirección Nacional Forestal-AID. Quito, Ecuador. 27p.

Kessler, M. \& P. Driesch. (1993). Causas e historia de la destrucción de bosques altoandinos en Bolivia. Ecología en Bolivia 21:1-18.

Kessler, M. (2000). Elevational gradients in species richness and endemism of selected plant groups in the central Bolivian Andes. Plant Ecology 149:181$193 \mathrm{p}$.

Lara, R. (1982). Contribuciones al conocimiento de la vegetación de las tierras altas de Bolivia. La Paz, Bolivia. $50 \mathrm{pp}$.

Loján, L. (2003). El Verdor de los Andes Ecuatorianos. SOBOC Grafic. Quito, Ecuador. 296 pp.

Mejía, F. (2011). Implicaciones ambientales del uso de leña como combustible doméstico en la zona rural de Usme. Tesis Mg. en Medio Ambiente y Desarrollo. Bogotá, Universidad Nacional de Colombia. Bogotá. $119 \mathrm{p}$.

MINAGRI, DGFFS \& PCM, OSINFOR (2012). Protocolo para la evaluación de individuos maderables. Lima.13 p.

MINAGRI-DGFFS. (2012). Manual base para la planificación y ejecución de inventarios forestales- 
-en bosques de producción permanente. Lima. 52 p.

MINAM. (2012). Memoria descriptiva del mapa de cobertura vegetal del Perú. Ministerio del Ambiente del Perú, Viceministerio de Desarrollo Estratégico de los Recursos Naturales, Dirección General de Evaluación, Valoración y Financiamiento del Patrimonio Natural. 76 p.

Moraes, M. Ollgaard, B. Kvist, L.P. Borchsenius, F. Balslev, H. (2006). Botánica económica de los andes centrales. Plural Editores. La Paz, Bolivia. 557 p.

Morlon, P. (1980). Plantar árboles es devolver la vida al Altiplano. ORDEPUNO. Convenio Perú-Canadá.

Ocaña, D. (1994). Desarrollo forestal campesino en la Región Andina del Perú. Ministerio de Agricultura, PRONAMACHS, FAO/HOLANDA. Talleres Gráficos D.C. Murakami S.A. Lima. 218 p.

ONERN, (1975). Mapa ecológico del Perú y memoria explicativa. Lima. 147 p.

Orme C. David L., Davies R. G., Burgess, Eigenbrod Felix, Pickup N, Olson V. A., Webster A. J., Tzung-Su Ding, Rasmussen P.C., Ridgely Robert S., Stattersfield, Ali J., Bennett P. M., Blackburn T. M., Kevin J. Gaston \& Ian P. F. Owens. (2005). Global hotspots of species richness are notcongruent with endemism or threat. Nature Publishing Group Vol 436. London.

Pedrotti, F., D. Gafta, A. Manzi \& R. Canullo (1992). Le associazioni vegetali della Piana di Pescasseroli (Parco Nazionale d'Abruzzo). Doc. Phytosoc. 14: 123-147.

Portuguez Y., H.; Matos D., D. \& Aucasime O., A. (2012). Memoria descriptiva del mapa de cobertura vegetal del Perú. Ministerio del Ambiente. Lima: 30$32 \mathrm{p}$.

Pretell, J. Chiclote, J., Ocaña Vidal, D., Jon Jap, R. \& Barahona Chura, E. (1985). Apuntes sobre algunas especies forestales de la sierra peruana-
- Proyecto FAO-Holanda/INFOR. Lima. 120 p.

IPrice, M.F., Gratzer, G., Alemayehu, L., Kohler, T., Maselli, D., Romeo, R. (2011). Mountain forests in a changing world - Realizing values, addressing challenges. FAO/MPS and SDC, Rome, Italy.

Reynel, C. (1990). Plantas para leña en el Suroccidente de Puno. Proyecto árbol andino. Puno, Perú. 165 pp.

Reynel, C. Pennington, T., Pennington, R., Marcelo, J. Daza, A. (2006). Arboles útiles del ande peruano. Talleres Gráficos de Tarea Asociación Gráfica Educativa. Breña, Lima. 462 pp.

Stadtmüller, T. (1986). Cloud forests in the humid tropics: a bibliographic review. The United Nations University and CATIE. Turrialba, Costa Rica.

Toivonen, J. \& Kessler. M. (2006). Distribución geográfica y los nichos ocupados de los bosques actuales de Polylepis en la Región del Cusco, Perú. II Congreso de Ecología y Conservación de Bosques de Polylepis. Cusco, Perú.

Walker, R. (1987). Land Use Transition and Deforestation in Developing Countries. Geographical Analisis. Vol. 19. pp. 18-30. https://doi.org/10.1111/j.15384632.1987.tb00111.x 